\title{
Etre à la fois scientifique et poète
}

\section{Both Scientist and Poet}

\author{
Georges Chapouthier ${ }^{1}$ \\ ${ }^{1}$ Directeur de Recherche Emérite au CNRS, georges.chapouthier@upmc.fr
}

RÉSUMÉ. Comment peut-on être à la fois scientifique et poète, deux activités a priori bien différentes, mais qui s'avèrent finalement complémentaires ? Le témoignage d'un chercheur scientifique, à la fois auteur de poèmes, lève une partie du voile sur cette question en montrant que la solution se trouve souvent dans la petite enfance. Le lien entre science et poésie peut ensuite être relayé par la complémentarité fonctionnelle des hémisphères cérébraux.

ABSTRACT. How can a person be both scientist and poet, carrying out two apparently different activities that may end up being complementary? The tale of a scientific researcher who also writes verse offers insights into the question and shows that such a reconciliation can often be traced back to early childhood. The link between science and poetry may then be extended and seen in the complementary functional roles of the two hemispheres of the brain.

MOTS-CLES. Biologie, Enfance, Hémisphères cérébraux, Morale, Poésie.

KEYWORDS. Biology, Childhood, Hemispheres of the brain, Morality, Poetry.

\section{Introduction}

Il peut, dans un premier temps, paraitre incongru de pratiquer à la fois une recherche scientifique et une écriture poétique, tant les deux univers semblent différents. La science doit se plier au creuset de la rationalité et de la vérité objective ; la poésie reste ouverte à tous les possibles et tous les imaginaires. La science adhère au réel ; la poésie peut emprunter les chemins les plus irréels ou les plus surréalistes. Pour le scientifique, la Terre est une planète bleue; pour le poète, elle peut être «bleue comme une orange », selon la magnifique formule d'Eluard.

Parvenu au seuil de ce qu'on appelle pudiquement le "troisième âge ", il m'a paru éclairant de retrouver, en partant de mes souvenirs d'enfance, des éléments qui ont pu contribuer à mes choix d'adulte et m'ont permis d'emprunter, à la fois, durant une cinquantaine d'années, ces deux voies si différentes de la rationalité scientifique et de l'imaginaire poétique. J'exposerai d'abord ces souvenirs lointains pour tenter ensuite de démêler leurs conséquences et de répondre, en confrontant parfois les deux domaines, à la question : comment peut-on être à la fois scientifique et poète ? ${ }^{1}$

\section{Tout se joue durant l'enfance}

Je suis né en France en 1945. C'est-à-dire que j'appartiens au «baby-boom » d'après-guerre, qui est devenu le « papy-boom », à la grande surprise des hommes politiques, qui n'avaient jamais imaginé qu'une marée de bébés pourrait donner, soixante-dix ans après, une marée de retraités ! Je suis né dans une famille aisée et intellectuelle, d'une mère charentaise très attachée à ses racines, et d'un père bordelais, professeur de Grec à Paris. On verra plus loin qu'il n'est pas sans importance de signaler que ma mère avait été l'étudiante de mon père, qu'elle était, d'une quinzaine d'années, plus jeune que lui. J'ai donc vécu ma petite enfance dans le milieu universitaire parisien, fier que mon père appartienne à ce que je considérais, du haut de mes trois pommes, comme l'élite du monde. Familiarisé dès le berceau aux dieux grecs, je buvais mon biberon sous les statues d'Athéna et de sa chouette. Un instant enfant unique, puisque aîné de la famille, je fus sur-choyé et appris à marcher et parler un peu plus tôt

\footnotetext{
${ }^{1}$ Une première ébauche, beaucoup plus réduite, de ce texte, est parue dans la Revue Indépendante (Paris), 2004, $\mathrm{n}^{\circ} 300$, pp 9-12, et dans la revue online Plastir, 2006, $\underline{5}$, 
qu'il n'est d'usage. Plusieurs évènements marquants vinrent cependant troubler cette enfance idyllique. Quand j'eus trois ans, je vis brusquement un bébé faire intrusion dans mon univers feutré. C'était ma petite sœur. Pour que je puisse débarrasser le plancher, on me mit alors, pour une année, au « jardin d'enfants » (on dirait aujourd'hui une « école maternelle » privée). Furieux, j'y allais tirer les cheveux de toutes les petites filles. En outre, et bien involontairement cette fois, j'y attrapai nombre de maladies infantiles - coqueluche, varicelle, angines...- que je passai aimablement à ma petite sœur. A la fin de l'année, mes parents décidèrent que, finalement, je serais mieux à la maison. Je retrouvai ainsi mon cocon protecteur et éducatif familial, dont on va mesurer l'importance sur les acquisitions de la petite enfance.

Quand j'eus cinq ans, des évènements apparemment indépendants rompirent mes habitudes. D'abord j'entrai en classe où $\mathrm{j}$ 'appris à lire. D'autre part, ma grand-mère paternelle mourut. Une vieille dame, que j'avais à peine connue, qui marchait avec difficulté entre la rue Notre-Dame-des-Champs et le jardin du Luxembourg, et à propos de laquelle ma mère émettait des propos amers que je comprenais mal. C'est aussi à cette époque que le père Noël eut la bonne idée de me donner un superbe ours en peluche, qui allait, des années durant, être mon animal fétiche.

Les deux derniers points, qui paraissent anecdotiques, appellent quelques réflexions. A la mort de ma grand-mère, un certain nombre d'objets arrivèrent en héritage. On me donna notamment des recueils de poésie « contemporaine » qui lui avaient appartenu. A l'âge où l'on commence à feuilleter des livres, j'y découvris avec passion des poèmes courts, à la métrique rigoureuse proche de la chanson - Paul Fort, Richepin, Maeterlinck...-, qui me marquèrent considérablement. Le goût prononcé de mon père pour les calembours, goût que j'acquis moi aussi à son contact, produisit également un intérêt précoce pour le jeu des mots et de leurs sonorités. La fibre littéraire venait de naître en moi.

\section{Une attirance pour les animaux}

Quant aux animaux, j'ai toujours eu pour eux une grande attirance. Tout petit, j'allais spontanément vers les chiens du village de Saintonge où je passai mes vacances avec ma mère et mes grands-parents maternels. Un peu plus tard, à l'âge où, à ce qu'on m'a appris, les petits garçons s'intéressent aux voitures et aux soldats, et les petites filles aux poupées, moi, je m'intéressais aux nounours. Et celui qui m'avait été donné par le père Noël, et qui allait devenir le fleuron d'une impressionnante collection, allait être un compagnon fusionnel de toute mon enfance.

Je crois d'ailleurs que certaines personnes, comme moi, ont une appétence très prononcée pour la forme des animaux quadrupèdes. J'ai retrouvé ce trait chez ma fille ainée. Alors qu'elle était toute petite, environ deux ou trois ans, est apparu à la télévision un dragon bavant et menaçant et, à ma grande surprise, $\mathrm{j}$ 'ai vu ma fille se précipiter vers le petit écran avec un mouvement de sympathie. Comme chez ma fille, la fibre de l'appétence animalière était bien présente, depuis toujours, au fond de moi.

\section{Approche des sciences et des premières versifications}

Un autre souvenir me revient : la joie de contempler avec mon père le ciel en Août, d'apprendre à reconnaître le premier et le dernier quartier de la lune, de savoir ce que sont les planètes, les étoiles... Ma décision fut prise ce soir-là : je serai astronome ! Mon père encourageait tous les désirs. Peu après, avec lui je rédigeai (c'est-à-dire qu'il rédigea pour moi) un mini « traité d'astronomie », qui me permit d'épater mes camarades de classe, par mes connaissances sur le soleil, les noms des planètes et leur différence avec les étoiles. Ma fibre scientifique venait de naître.

Comme je m'intéressais aux poètes préférés de ma grand-mère et aux rimes, mon père rédigea aussi pour moi un poème régulier de trois quatrains, comportant les trois organisations possibles des rimes : abba, abab et aabb. Il était question d'une baignade suivie d'un risque de noyade et seuls les deux 
dernières strophes me sont restées en mémoire (mille fois pardon, lecteur éduqué, pour ces vers de mirlitons, évidemment adaptés par mon père à un bambin de cinq ans !) :

... Alors mon ours joli

En me voyant noyer

A fait un clapotis

Pour venir me sauver.

Et nous rentrons tous deux

En contemplant les cieux

Jusqu'à notre maison

Où nous nous reposons.

Si j'ai tenu à citer cet humble souvenir de la petite enfance, c'est que, sans que je m'en rende compte, il portait sans doute en lui mon attrait ultérieur pour la poésie en général et pour certaines règles de métrique en particulier. Je souligne donc ici l'importance de la formation précoce du bambin à ce que seront les intérêts de l'adulte.

\section{Une grande rupture}

La mort de mon père, alors que j'avais huit ans, fut un cataclysme dont je ne me suis jamais vraiment remis. Ma mère dut prendre un double visage, difficile à assumer, à la fois maternel et paternel. Des parents, des amis, me vinrent aussi en aide : un grand-père maternel médecin de campagne et collectionneur de papillons, un oncle et une tante pharmaciens, un ami de mon père, Henri Seyrig, (le père de l'actrice Delphine Seyrig), archéologue mais très ouvert sur la biologie... Quand j'eus treize ans, je passais plusieurs mois chez les Seyrig, à Beyrouth au Liban. J'y découvris le jeu d'échecs, le multiculturalisme, l'odeur enivrante des citronniers en fleurs, la peinture abstraite, enfin la poésie de l'écrivain libanais de langue française Georges Schéhadé et une manière non rationnelle d'utiliser les mots. Derrière la fleur, me disait, en substance, Schéhadé, il n'y a pas un singe, mais un enfant, le visage en pleurs. A l'époque, jeune garçon plutôt rationnel, j'eus beaucoup de mal à comprendre et à assimiler l'esprit de ce message, qui me paraissait tout à fait illogique. Mais, amplifié par les découvertes ultérieures des Symbolistes et des Surréalistes, il devait en fait me suivre toute ma vie.

\section{Des études scientifiques}

Quand il fallut choisir des études, mon goût pour les sciences, pour la nature, et particulièrement pour les animaux, me poussa vers la biologie. Le milieu dont j'étais issu me fit naturellement préparer les Grandes Ecoles et, avec la biologie pour guide, je me retrouvai au sein d'une carrière toute tracée : l'Ecole Normale Supérieure en biologie, puis le CNRS, où je passai plus d'une quarantaine d'années à effectuer, sur des souris, des recherches en neuropharmacologie ${ }^{2}$.

Mais, en parallèle, me hantaient des éléments importants depuis mon enfance et auxquels la biologie ne répondait pas vraiment. Depuis toujours, mon intérêt scientifique pour les animaux se doublait en effet d'une affection pour eux. Or la biologie conduisait parfois à leur faire des misères. Bien sûr, c'était permis, c'étaient des misères jugées absolument légitimes ! Comme l'avaient dit Descartes ${ }^{3}$ et Malebranche. Comme on me l'avait répété toute mon enfance, aussi bien à l'école laïque qu'au

${ }^{2}$ G. Chapouthier et F. Tristani-Potteaux, Le chercheur et la souris, CNRS Editions, Paris, 2013.

${ }^{3}$ G.Chapouthier, Impact de l'animal-machine sur la biologie moderne : tromphe épistémologique et désastre moral, $L^{\prime}$ esprit cartésien, Actes du XXVI ${ }^{\circ}$ Congrès de l'association des Sociétés de Philosophie de Langue Française (ASPLF), Paris 30 Août-3 Septembre 1996, Vrin éditeur, Paris, 2000, Tome II, pp 742-744. 
catéchisme : l'homme avait, sur la bête, tous les droits. Tous les droits. Y compris celui de tirer sur les écureuils, comme s'en était vanté, durant mon enfance, le coiffeur de mon village en Charente Maritime, chasseur passionné, devant son public de clients admiratifs et amusés. Y compris celui d'abattre les animaux pour les manger, comme cette jeune vache aperçue là où, gamin, je n'aurais jamais dû être, dans un abattoir artisanal des Pyrénées. Cette jeune vache qui, traînée dans les flots de sang de la précédente victime, me regardait fixement, moi, enfant comme elle. Y compris celui d'asphyxier les papillons à la benzine, pour les épingler dans des cadres où ils pourriront quelques années plus tard. De la superbe collection de mon grand-père, tout est finalement tombé en poussière.

Ces souvenirs amers, en même temps qu'une réflexion sur ma discipline, la biologie, science des systèmes complexes, me conduisirent à la philosophie, à laquelle j'ai consacré de nombreux livres, tant sur la complexité du vivant ${ }^{4}$ que sur les droits de l'animal ${ }^{5}$.

\section{Deux activités contradictoires ?}

Quant à la poésie, depuis le Maeterlinck de mes cinq ans, elle n'a jamais cessé de m'accompagner. J'ai conscience d'être d'abord un rêveur. Ensuite seulement je suis devenu un « savant », ou plutôt un poète égaré dans la science et qui $\mathrm{y} a$, très modestement, réussi ${ }^{6}$. Mais $\mathrm{j}$ 'ai toute ma vie effectué des travaux d'ordre poétique. Pour éviter les ennuis professionnels ou les reproches qui auraient pu m'être faits par les commissions du CNRS sur cette double activité, apparemment contradictoire, j'ai pris, lors de mon séjour pré-doctoral à l'Université de Strasbourg, le voile d'un pseudonyme alsacien, Georges Friedenkraft ${ }^{7}$, destiné à couvrir mes activités d'ordre poétique ou littéraire. C'est ainsi que, sous ce pseudonyme, j'ai écrit d'innombrables textes littéraires, parfois dans de grands journaux comme Le $M o n d e^{8}$, le plus souvent dans des revues de création à faible tirage, et que j'ai participé à des comités de rédaction de revues littéraires. La participation plus importante a été mon activité, de 1984 à 2016, au comité de lecture et de rédaction de la revue Jointure ${ }^{9}$, dont j'ai fini président de l'association éditrice «La Jointée ». Cette revue, d'une rare longévité parmi les revues de poésie, dut cesser sa parution en 2016 avec son numéro symbolique 100, suite au décès de plusieurs autres de ses animateurs ${ }^{10}$.

Recherche scientifique et activité poétique entrent parfois en compétition. En témoignent deux forfaits involontaires, deux journées qui auraient dues être consacrées exclusivement à la science, et que la poésie a partiellement détournées de leur fin première. Le jour où, grâce au regretté Jacques Arnold, je découvris le «monostiche » ou poème en seul vers. Le jour où, grâce à Haruki Majima, de Tokyo, je découvris le « haïkou » (en général écrit « haïku», mais dont j’aime bien franciser le nom ${ }^{11}$ ) et son rythme traditionnel : trois vers de cinq, sept et cinq pieds respectivement. Dans les heures qui suivirent, ma tête se mit à travailler sans arrêt sur ces formes, nouvelles pour moi, et me fit accoucher,

\footnotetext{
${ }^{4}$ G.Chapouthier, L'homme, ce singe en mosä̈que, Préface de Patrick Blandin, Editions Odile Jacob, Paris, 2001.

${ }^{5}$ G.Chapouthier, Qu'est-ce que l'animal ?, Collection "Les petites pommes du savoir", Editions le Pommier, Paris, 2004 ; G.Chapouthier, J.C.Nouët (editors), The universal declaration of animal rights, comments and intentions, Editions Ligue Française des Droits de l'Animal, Paris, 1998.

${ }^{6}$ Pour ce bilan, voir aussi : G. Chapouthier et F. Tristani-Potteaux, Le chercheur et la souris, CNRS Editions, Paris, 2013.

${ }^{7}$ https://fr.wikipedia.org/wiki/Georges_Friedenkraft. Le mot signifie en alsacien « la force de la paix », tout un programme !

${ }^{8}$ G. Friedenkraft, Mais où est passée Athénes ? (témoignage), Le Monde, Samedi 17 Novembre 1979, N ${ }^{\circ}$

$10824,21$.

${ }^{9}$ https://fr.wikipedia.org/wiki/Jointure_(revue_littéraire).

${ }^{10}$ G. Friedenkraft, Editorial : « Bon cent : nous y voilà ! », Jointure, 2016, 100, 3-4.

${ }^{11}$ G. Friedenkraft, Style et esprit des haïkou en français, Bulletin des Anciens Elèves de l'INALCO, Avril 2002, 113-120. 
sans que je le demande, de cascades de monostiches et de marées de haïkous ! Mes travaux au laboratoire en furent quelque peu perturbés, car, au détour de chaque problème scientifique ou technique que je devais me poser, un poème surgissait de façon impérative et m'obligeait à en marteler le rythme ou la mélodie. Comme quoi, quand le rythme et la métrique vous tiennent, il est bien difficile de s'en abstraire! Heureusement aucun de mes collègues scientifiques n'a jamais su que, tout en dévissant une prise de courant ou en nettoyant la cage d'une souris, j'étais ainsi obsédé par des formes poétiques dont je n'arrivais pas à me débarrasser !

Une autre contradiction, plus amusante celle-ci, a été fournie par une aventure survenue dans une galerie de peinture ouverte, rue Saint-Jacques à Paris, près de chez moi, par une artiste vietnamienne. Sur cette galerie, "La Sensitive », j'avais écrit, dans un périodique local et sous mon pseudonyme, un article élogieux. Lors d'un vernissage avaient été prises des photos où je figurais. Un jour la directrice de la galerie montra ces photos à deux habitantes de mon immeuble en leur disant :

- Voici Monsieur Friedenkraft qui a écrit ce bel article sur ma galerie !

- Mais non, répliquèrent, presque outragées, mes deux voisines d'immeuble ! Vous vous trompez : il s'agit de Monsieur Chapouthier, un chercheur scientifique qui habite à notre adresse !

Elles en vinrent presque aux mains, tant elles étaient convaincues, les unes comme l'autre, et pour cause, d'avoir parfaitement raison.

Et la question alors ? Comment devient-on à la fois scientifique et poète ? On remarquera d'abord qu'une large part de la réponse réside dans l'endroit où l'on est né. Et puisque c'est une bénédiction d'être poète (je suis moins affirmatif sur le fait d'être scientifique), pour ce point au moins il faut être né chanceux, avec un père qui aime regarder les étoiles autant que jouer avec les mots, et une mère prête, en cas de coup dur, à consacrer toute sa vie à l'éducation de ses enfants. Une large part de la réponse réside en fait dans la formation et les acquisitions de la petite enfance.

\section{Ethique animalière et poésie : une même voie?}

Ce n'est pas un secret que l'être humain possède deux hémisphères cérébraux aux fonctions différentes et complémentaires ${ }^{12}$. Schématiquement, chez le sujet standard droitier (les choses sont plus compliquées chez les gauchers), l'hémisphère gauche est responsable de la pensée analytique et abstraite, et l'hémisphère droit de la pensée synthétique et concrète. On aurait donc d'abord tendance à associer la poésie et la science, qui reposent toutes les deux sur le discours et le langage, à l'hémisphère gauche, et la morale, porteuse de valeurs plus globales, à l'hémisphère droit. Mais ce n'est pas exactement le cas. Certes la science repose largement sur l'activité analytique et rationnelle de l'hémisphère gauche. Mais si la poésie, dans l'articulation de ses mots, est effectivement aussi un morceau de discours et de langage, lié à l'hémisphère gauche, les sentiments qui la guident, le contenu émotionnel et existentiel qu'elle véhicule, la rend très proche d'un sens des valeurs, donc parente à certains égards, de la perception spirituelle et morale. Dans ce cas, l'intérêt qui m'a poussé à la réflexion morale sur les animaux n'est alors peut-être pas étranger à ma passion de toujours pour la poésie.

Si l'on suit ce raisonnement schématique, être scientifique et poète relèverait finalement d'une bonne harmonie, acquise durant l'enfance, d'une complémentarité complice entre les deux hémisphères cérébraux. Quant à la liberté là-dedans, elle reste bien cachée, et se trouve peut-être dans la manière très personnelle que nous avons chacun de regarder les mêmes étoiles et les mêmes animaux.

\footnotetext{
${ }^{12}$ Sur ce point, voir notamment G. Chapouthier, Kant et le chimpanzé-Essai sur l'être humain, la morale et l'art, Editions Belin, Paris, 2009 ; Prix « Achille Urbain » 2010 de l'Académie Vétérinaire de France. 


\section{En guise de conclusion}

Sans doute devrais-je conclure cette réflexion sur les deux disciplines évoquées - biologie et poésie - par un poème consacré aux animaux ${ }^{13}$ :

Belle vampire

J'ai besoin de sang

(et je m'en excuse)

je suis ainsi faite

Mais que seraient sans

ce sang mes pommettes

ma taille fine et

mes jambes de fée?

que seraient mon charme

et ma silhouette?

Je serai discrète

j'attendrai, bel homme, ton sommeil de plomb

à l'heure où le rêve

fou te désarçonne

vers Eros pervers

pour étancher ma

soif-pardonne-moi

je suis ainsi faite

Je sais, beau bipède

que je te ferai

souffrir de ma bouche

(et j'en suis confuse)

mais sous les reflets

d'argent de la lune

je te saignerai

je suis ainsi faite

homme succulent!

mon superbe athlète!

moi fille de l'air

moi belle diptère

Moustique je suis

et l'amour en tête !

${ }^{13}$ G. Friedenkraft, Sur les sentiers du songe (poèmes pour mettre la vie en musique), Prix Robert-Hugues Boulin, Editions Sajat, Paris, 2015, p 40. 